Escuela de Kinesiología, Facultad de Medicina, Universidad de Chile.

Equipo de Rehabilitación Respiratoria, Clínica Los Coihues.

${ }^{3}$ Facultad de Ciencias de la Salud Blanquerna, Grupo de Investigación en Fisioterapia (GReFis), Universidad Ramón Llull. Barcelona, España. aKinesiólogo.

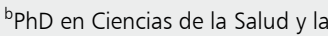
Vida, UPF Barcelona.

Recibido el 19 de abril de 2013, aceptado el 20 de enero de 2014.

Correspondencia a: Rodrigo Torres-Castro. Av. Independencia 1027 Santiago, Chile. klgorodrigotorres@gmail.com

\section{Estrategias terapéuticas para aumentar la eficacia de la tos en pacientes con enfermedades neuromusculares}

\author{
RODRIGO TORRES-CASTRO ${ }^{1,2, a}$, GONZALO MONGE 2, , \\ ROBERTO VERA ${ }^{1, \mathrm{a}}$, HOMERO PUPPO ${ }^{1, \mathrm{a}}$, \\ JUAN CÉSPEDES ${ }^{2}$, JORDI VILARÓ

\section{Therapeutic strategies to increase the effectiveness of cough}

\begin{abstract}
Cough is a natural reflex that protects respiratory airways against infections or mucus retention. Cough maintains an adequate cleaning of the airways and is a mainstay of respiratory therapy. It can be triggered voluntarily by the patient or by a specific cough device. Peak cough flow (PCF) is used to assess the effectiveness of the cough. When this value is below $160 \mathrm{~L} / \mathrm{min}$, cough is considered inefficient and becomes a risk factor for respiratory problems. Patients with weak cough, especially those with neuromuscular disease, have in common a low tidal volume and a decreased maximum insufflation capacity. Both factors directly affect the inspiratory phase previous to cough, which is considered vital to obtain the optimum flow for a productive cough. Different therapeutic measures may help to increase cough efficiency among patients with cough weakness. These interventions may be performed using manual techniques or by mechanical devices. The aim of this review is to analyze the different techniques available for cough assistance, set a hierarchy of use and establish a scientific basis for their application in clinical practice.
\end{abstract}

(Rev Med Chile 2014; 142: 238-245)

Key words: Cough; Forced expiratory flow rates; Neuromuscular Diseases.
L a tos en un reflejo natural de protección de la vía aérea que cumple con dos funciones fundamentales: mantener las vías respiratorias libres de elementos extraños y expulsar el exceso de secreciones producidas en condiciones reológicas anormales ${ }^{1}$. Si bien la tos se define como un reflejo, puede ser desencadenada de forma voluntaria por el paciente o provocada por un profesional constituyendo una herramienta importante dentro del arsenal terapéutico destinado a mantener un adecuado drenaje de secreciones. Es utilizada en una gran variedad de patologías, principalmente en adultos que colaboren pero, en circunstancias específicas, puede ser desencadenada de forma involuntaria en lactantes, pacientes con daño neurológico o cualquier enfermo no colaborador ${ }^{2}$.
El objetivo de este texto fue analizar los mecanismos fisiológicos de la tos, las herramientas para evaluar su eficacia y clasificar las distintas técnicas manuales e instrumentales que actualmente el terapeuta tiene a su disposición para procurar el mayor beneficio al paciente.

\section{La tos}

Clásicamente, la literatura describe tres fases en la ejecución de la tos: fase inspiratoria, que consiste en inspirar el mayor volumen de aire posible; fase compresiva, que comprende el cierre de la glotis y la presurización del sistema respiratorio esencialmente por la contracción de la musculatura 
abdominal $^{3}$; por último, la fase expulsiva, donde se produce una expulsión violenta del volumen al mayor flujo posible ${ }^{4}$. Algunos textos refieren la presencia de una cuarta fase, previa a las demás, llamada fase irritativa, fundamentalmente pensando en una tos involuntaria ${ }^{5}$. Esta consiste en la estimulación de receptores tusígenos a nivel de tráquea o bronquios proximales ${ }^{3}$.

La fase más importante de la tos, y la que determina en gran parte su eficacia, es la fase inspiratoria $^{6}$. Trebbia y cols, en un estudio realizado en 155 pacientes con enfermedad neuromuscular, analizaron la correlación existente entre el pico flujo de tos (PFT) y parámetros de función pulmonar evaluados con espirometría y ventilometría. Estos autores demostraron que los factores que más influyen en la eficacia de la tos son la capacidad vital (CV), la capacidad de insuflación máxima (CIM) y la presión inspiratoria máxima (PIM), relegando a un papel secundario parámetros espiratorios como la presión espiratoria máxima (PEM), entre otros ${ }^{6}$.

\section{Procedimiento para la evaluación de la tos}

La evaluación de la tos se realiza a través del PFT mediante un flujómetro portátil, dispositivo mecánico utilizado comúnmente para la evaluación del flujo espiratorio máximo (FEM), o un neumotacómetro, dispositivo electrónico utilizado habitualmente en la realización de espirometrías ${ }^{7}$. La interfase utilizada puede ser una mascarilla facial ${ }^{8}$ o una boquilla ${ }^{9,10}$. Existe controversia en cuál es la mejor interfase para realizar esta evaluación. Trebbia y cols observaron que la fuga de aire fue marcadamente menor cuando se usaba mascarilla en lugar de boquilla ${ }^{6}$. Otros autores, sugieren utilizar boquilla excepto en aquellos que tengan debilidad de la musculatura bucal ${ }^{11}$ que impida un cierre correcto de los labios sobre la boquilla, lo que implicaría una fuga de aire que puede provocar una caída en el flujo y, por lo tanto, una subestimación de la eficacia de la tos.

La literatura describe que el volumen movilizado en el acto de toser debe ser al menos de 2,3 $\pm 0,5$ litros con un flujo comprendido entre 6 y $20 \mathrm{~L} / \mathrm{s}^{4,12}$. Por lo tanto, para poder obtener un PFT eficaz, es necesario realizar una inspiración inicial mayor a 85-90\% de la CIM y una presión toracoabdominal superior a $100 \mathrm{cmH}_{2} \mathrm{O}^{12,13}$. Cuando los valores de flujo espiratorio obtenidos son menores a $160 \mathrm{~L} /$ min, se consideran ineficaces para la eliminación de secreciones ${ }^{9}$. En tanto, valores menores a 270 $\mathrm{L} / \mathrm{min}$, generan un mayor riesgo en el desarrollo de complicaciones respiratorias, lo cual fue demostrado en pacientes adultos con distrofia muscular de Duchenne (DMD) ${ }^{14}$ y refrendado posteriormente por Tzeng en otras patologías neuromusculares ${ }^{15}$.

Con el conocimiento actual, podemos establecer un método de evaluación de la eficacia de la tos en cuatro etapas, que permita determinar la necesidad de asistir la tos (Figura 1). En la primera etapa debe realizarse una medición del PFT. En función de los valores obtenidos, en la segunda etapa procederemos a evaluar la CV, la cual determinará, conjuntamente con el PFT, si en la tercera etapa el paciente ingresa al protocolo de tos asistida. En la cuarta etapa, determinaremos qué técnica emplear en función de: integridad de la vía aérea (cierre de glotis), colaboración del paciente y tipo de soporte ventilatorio que utiliza.

\section{Debilidad de la tos y patología}

Existe un importante número de pacientes que presentan afectación de la tos. Entre éstos, los que padecen enfermedades neuromusculares ocupan un lugar relevante ${ }^{16}$. Este tipo de patologías, se caracteriza por una debilidad progresiva que afecta la musculatura esquelética, respiratoria, deglutoria, y en algunos casos cardiaca ${ }^{17}$. La ineficacia de la tos y la dificultad de remover secreciones es una característica común a estas enfermedades y es causante de la gran prevalencia de morbimortalidad respiratoria ${ }^{14,17}$.

Los pacientes con enfermedad neuromuscular movilizan un menor volumen corriente (VC) producto de la debilidad severa de su musculatura respiratoria. La pérdida de contractibilidad muscular conlleva a que exista una disminución de la movilidad de la caja torácica, lo que determina menores rangos de movimiento y alteraciones de las propiedades elásticas del pulmón, afectando finalmente la distensibilidad toracopulmonar ${ }^{18}$. Además, la disfunción bulbar dificulta el cierre y apertura rápida de la glotis durante la tos, incluso en presencia de una función normal de la musculatura respiratoria ${ }^{19}$.

Por otro lado, en pacientes con esclerosis lateral amiotrófica (ELA) ${ }^{20}$ y DMD ${ }^{21}$, la fuerza muscular inspiratoria y espiratoria están disminuidos de 


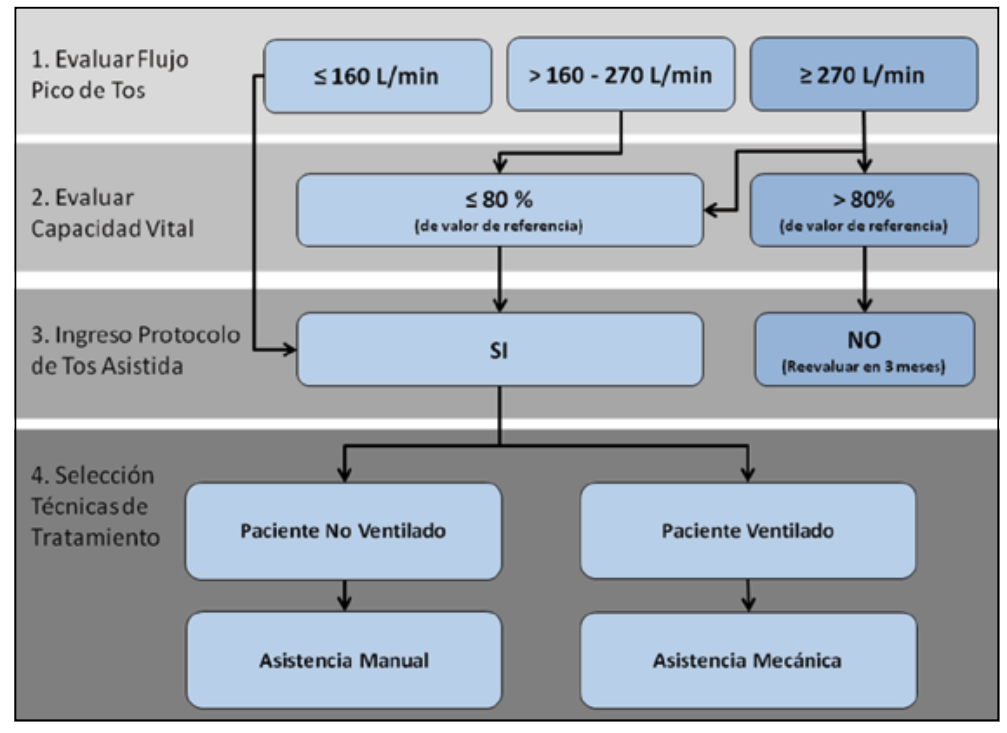

Figura 1. Algoritmo de evaluación de la tos. forma similar. Esto va en detrimento tanto de la fase inspiratoria como de la fase expulsiva de la tos. En otras afecciones, como la atrofia muscular espinal (AME), la debilidad muscular espiratoria es la predominante ${ }^{22}$. Por último, otra patología que presenta una gran afectación de la tos, es la lesión medular ${ }^{23}$, lo que se refleja en que esta población fallece principalmente por causa respiratoria, siendo la neumonía una de las principales patologías que producen la muerte durante el primer año post lesión ${ }^{24}$. La musculatura espiratoria se afectará de manera variable si la lesión se encuentra por encima de L3, involucrando principalmente la musculatura abdominal y la musculatura inspiratoria. Las lesiones por encima de T11, afectaran a los intercostales internos; sobre C8 a los escalenos y por encima de C5, al diafragma, motor fundamental de la musculatura inspiratoria ${ }^{25}$. El nivel de lesión medular tiene relación con la alteración que sufren las variables espirométricas, por ejemplo, la capacidad vital forzada (CVF). Esto fue estudiado por Lin y cols en 2001, estableciendo como punto crítico el nivel T7-T8 ya que lesiones por encima de este nivel disminuirán la CVF en $20 \%$ pudiendo llegar incluso, a 50\% cuando la lesión es a nivel C2 o C $3^{25}$. En otro trabajo, Spungen determinó que además de producirse una disminución de $25 \%$ del VC, se producía una caída de 75\% en el número de suspiros en los lesionados medulares altos versus controles sanos, afectando directamente el fenó- meno de hiperinsuflación periódica espontánea ${ }^{25}$. En consecuencia, el nivel de lesión es el factor más importante que determina la morbimortalidad respiratoria.

\section{Asistencia de la tos}

En pacientes que presentan alteraciones de la tos, existen variadas alternativas terapéuticas que permiten incrementar la eficacia de cada una de sus fases. La asistencia puede ser realizada mediante técnicas manuales o con la ayuda de dispositivos mecánicos que serán seleccionadas en función de la evaluación específica realizada y considerando la fase sobre la cual actúan. Para facilitar la comprensión del lector, clasificaremos las técnicas de asistencia de la tos agrupándolas en dos categorías (Figura 2).

\section{Asistencia manual de la tos}

La eficacia de la tos está directamente relacionada con el volumen inspiratorio previo a la maniobra expulsiva ${ }^{6}$. Para ello, en el caso que el volumen obtenido no sea el adecuado, es necesario introducir una maniobra inspiratoria asistida previa. Desde la perspectiva del terapeuta respiratorio, esta forma parte de la asistencia de la tos, ya que de ella depende directamente su eficacia. En consecuencia, hemos establecido una jerarquización 


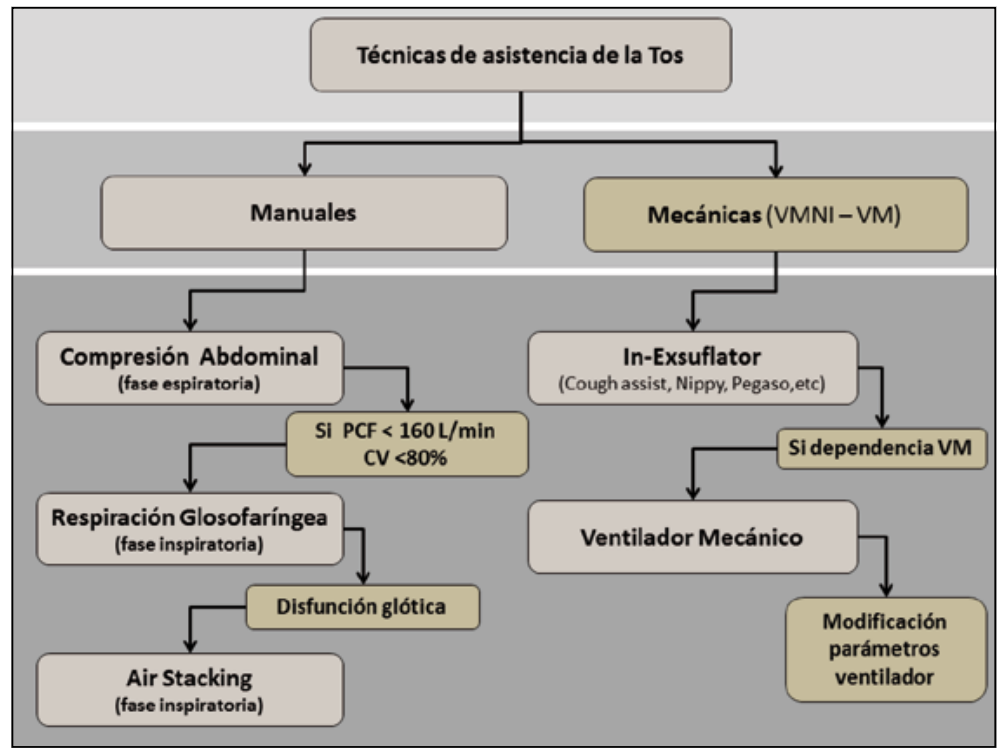

Figura 2. Técnicas de asistencia de la tos. de las técnicas, basado principalmente en el nivel de dificultad de la realización de las técnicas, de menor a mayor complejidad y por orden de menor a mayor afectación según el grado de disfunción.

a) Compresión abdominal: Esta técnica tiene como objetivo favorecer la fase expulsiva de la tos. Consiste en colocar las manos en la parte superior del abdomen y ejercer una presión en sentido posterior y cefálico durante la espiración. Este tipo de asistencia también es conocida como tos cuadripléjica ${ }^{16}$ y ha sido descrita también con compresión torácica o compresión toracoabdominal. En una investigación con 28 pacientes con DMD con $\mathrm{CVF}<$ a $60 \%$ del predicho, la técnica ha demostrado aumentar los valores de PFT por encima de los $160 \mathrm{~L} / \mathrm{min}^{26}$, demostrando cambios significativos en esta población. Braun y cols reportaron, en una serie de 13 sujetos con lesión medular entre $\mathrm{C} 4$ y $\mathrm{C} 6$, que el PFT aumentaba significativamente $13,8 \%$ en promedio respecto a la tos no asistida $^{27}$.

b) Respiración Glosofaríngea (RGF): Descrita en 1951 por Clarence Dail ${ }^{28}$. Esta técnica actúa sobre la fase inspiratoria de la tos. Consiste en tomar múltiples insuflaciones mediante movimientos de la boca, mejillas, lengua, faringe y laringe para conseguir "tragar aire" enviándolo hacia los pulmones ${ }^{28}$. El objetivo es substituir la musculatura inspiratoria débil por la acción de la musculatura orofaríngea. Para que la técnica sea eficaz, se debe contar con indemnidad de la glotis y debe acompañarse de un buen aprendizaje de la maniobra por parte del paciente. Para obtener un volumen final superior al volumen corriente, cada insuflación debe suponer al menos unos 80 $\mathrm{mL}$. Nygren-Bonnier, sometió a 25 sujetos con lesión medular cervical a una serie de 10 ciclos de RGF cuatro veces a la semana durante 8 semanas, mejorando significativamente su CV en $25 \%{ }^{29}$. Posteriormente, estos autores, en un grupo de 11 niños con AME tipo II a quienes aplicaron el mismo protocolo utilizado con los lesionados medulares, demostró efectos positivos de la RGF, incrementando la CV, FEM y la expansión torácica de manera significativa ${ }^{30}$.

c) Estancamiento de aire o Air Stacking (AS): Esta técnica actúa durante la fase inspiratoria. Consiste en entregar múltiples insuflaciones de aire a través de una bolsa de reanimación manual, buscando alcanzar la CIM $^{11,26}$. Esta técnica aumenta el volumen inspirado y remplaza las insuflaciones periódicas (suspiros), contribuyendo además, a mejorar la movilidad torácica y prevenir atelectasias. La CIM es un indicador indirecto de distensibilidad pulmonar. La técnica 
clásica está descrita con una bolsa de reanimación manual, una válvula unidireccional, un corrugado entre 20 y $30 \mathrm{~cm}$ y en el caso que exista fuga de aire, una pinza nasal ${ }^{31}$. Su mayor eficacia, se ha reportado en pacientes con enfermedades neuromusculares y lesionados medulares ${ }^{10,32}$. En un estudio con 28 pacientes con DMD con CVF $<$ a $60 \%$ del predicho, ventilados mecánicamente de forma no invasiva durante la noche, el PFT basal aumentó significativamente desde $171 \pm 67 \mathrm{~L} / \mathrm{min}$ hasta $225 \pm 80 \mathrm{~L} / \mathrm{min}^{26}$. Cuando la maniobra era acompañada con compresión torácica durante la fase espiratoria, el PFT alcanzó los $292 \pm 86 \mathrm{~L} /$ min. En otro estudio con 61 pacientes con DMD, de los cuales 34 usaban soporte ventilatorio, la tos no asistida fue de $138 \pm 70 \mathrm{~L} / \mathrm{min}$ y aumentó significativamente hasta $302 \pm 78 \mathrm{~L} / \mathrm{min}$ en el grupo sometido a AS más asistencia espiratoria manual abdominal ${ }^{33}$. Observándose más beneficios en los pacientes que presentaban mayor debilidad de la tos (aquellos que en la evaluación tenían un PFT $<90 \mathrm{~L} / \mathrm{min}$ ). En algunos artículos, se describe que el AS se puede realizar con un ventilador controlado por volumen ${ }^{10}$.

Cada una de las técnicas antes mencionadas, aplicadas por separado, contribuyen a mejorar la tos, pero la combinación de éstas hace que la tos sea aun más efectiva ${ }^{16,26}$. La evidencia nos muestra que al realizar AS combinado con compresión abdominal manual, se logran niveles de PFT que incluso pueden alcanzar valores de normalidad, hecho que podría explicarse por qué el AS favorece la fase inspiratoria y la compresión manual la fase espiratoria ${ }^{26}$.

\section{Asistencia mecánica de la tos}

Actualmente, existe una gran variedad de dispositivos que permiten de forma pasiva o con la colaboración del paciente, mejorar mecánicamente las fases inspiratoria y/o espiratoria de la tos. Cómo no todos los equipos actúan por igual, es necesario conocer sus principios de operación para determinar el uso más apropiado en cada paciente y/o situación.

a) Insuflador-exsuflador: Consiste en entregar, mediante un dispositivo mecánico, una insuflación profunda seguida de una exhalación forzada, simulando una tos natural ${ }^{12}$. Es generada por un equipo que entrega presión positiva inspiratoria y negativa espiratoria (efecto succión) de al menos
+40 a $-40 \mathrm{cmH}_{2} \mathrm{O}^{34}$. Puede conectarse al paciente a través de una interfase bucal, nasobucal o una traqueostomía. Fue descrita inicialmente por Alvan Barach en 1953 en pacientes con poliomelitis ${ }^{35}$ y gracias a los buenos resultados obtenidos, se comenzaron a diseñar dispositivos, primero con presión negativa y luego con presión positiva, buscando optimizar la mecánica de la tos. Entre ellos podemos mencionar el Cough Assist In-exsufflator (Respironics Corporation. Palo Alto, USA), Pegaso (Dima. Bolonia, Italia), y el Nippy Clearway (B\&D Electromedical. Warwickshire, Inglaterra). Su eficacia ha sido demostrada en numerosas investigaciones $8,12,19,36,37$. En un estudio realizado en 22 pacientes con enfermedades neuromusculares e historia de exacerbaciones respiratorias recurrentes se constató incrementos significativos de PFT desde $169 \pm 90 \mathrm{~L} / \mathrm{min}$ hasta $235 \pm 111 \mathrm{~L} / \mathrm{min}$ luego de seguir un protocolo de asistencia mecánica de la tos con el Cough Assist programado en +40 a $-40 \mathrm{cmH}_{2} \mathrm{O}^{8}$. Gonçalvez y cols, en un estudio aleatorizado, compararon dos grupos de pacientes conectados a ventilación mecánica convencional a través de un tubo endotraqueal, en una unidad de cuidados intensivos que cumplieran con criterios para extubación. Un grupo utilizó 3 sesiones con Cough Assist desde +40 a $-40 \mathrm{cmH}_{2} \mathrm{O}$, con 8 ciclos por sesión, antes y después de la extubación, y se comparó con un grupo con tratamiento convencional que consistía en oxígeno suplementario, broncodilatadores, antibióticos y ventilación no invasiva. El grupo intervenido tuvo un éxito de $48 \%$ en la extubación en comparación con $17 \%$ del grupo control y estuvo menor número de días conectado a ventilación mecánica (12d) en comparación del grupo control $(18 \mathrm{~d})^{37}$, siendo estos resultados estadísticamente significativos.

Algunos autores han sugerido que el uso de asistencia mecánica de la tos puede disminuir o evitar la necesidad de succión de secreciones en pacientes con enfermedades neuromusculares, ya que el PFT que se obtiene es suficiente para eliminarlas, evitando los efectos deletéreos de la succión repetitiva en la mucosa de la vía aérea ${ }^{12}$. Por último estos equipos, por su elevado costo, no están disponibles para todos los profesionales. Sin embargo, es posible su uso entre distintos pacientes, siempre y cuando se respeten los estrictos niveles de limpieza y esterilización de circuitos, similares a los aplicados para los equipos de ventilación mecánica. 
b) Ventilación mecánica como dispositivo de tos: Consiste en asistir la tos utilizando un ventilador mecánico controlado por volumen o un generador de flujo. Siguiendo los mismos principios fisiológicos ya comentados, se aumenta el volumen inspirado y el flujo espirado para reproducir los mecanismos de la tos natural.

Esta técnica fue evaluada en un estudio de 179 pacientes con enfermedad neuromuscular ${ }^{16}$, en los que se comparó los efectos en el PFT sin asistencia de la tos, tos asistida mediante ventilador controlado por volumen y tos asistida con ventilador más compresión abdominal manual. Los resultados mostraron que el grupo no asistido tuvo un PFT promedio de $108 \mathrm{~L} / \mathrm{min}$, el grupo con tos asistida con VM obtuvo un PFT de $202 \mathrm{~L} /$ min y el PFT de la maniobra combinada fue de $248 \mathrm{~L} / \mathrm{min}$, demostrando una mayor eficacia cuando se combinaron las dos técnicas. Además, el uso del ventilador como asistente de la tos junto con compresión manual ha reportado disminuir el número de hospitalizaciones asociadas a infecciones respiratorias altas $^{38}$. Senent y cols, en una investigación de 28 pacientes con ELA con soporte ventilatorio no invasivo, comparó diferentes técnicas de asistencia de la tos, utilizando tos asistida con ventilador mecánico no invasivo en modalidad binivel con los parámetros que utilizaba habitualmente el paciente, otro grupo con la misma modalidad pero con un IPAP de $+30 \mathrm{cmH}_{2} \mathrm{O}^{39}$. Ambos grupos mostraron mejorar significativamente el PFT, no existiendo diferencias significativas entre aquellos con y sin compromiso bulbar. Al igual que el AS esta técnica permite mantener la distensibilidad torácica y simular los suspiros fisiológicos que en pacientes conectados a ventilación mecánica están disminuidos o abolidos, aumentando el riesgo de atelectasias. Recientemente se ha desarrollado un nuevo ventilador mecánico domiciliario (VENTIlogic ${ }^{\circledast}$ LS Weinmann Geräte für Medizin $\mathrm{GmbH}$. Hamburg, Alemania) que dispone de una función de asistencia de la tos incorporada a los modos ventilatorios, denominada LIAM $^{\circledast}$ (Lung inflation assist manoeuvre). Esta función consiste en insuflar los pulmones con una presión alta que puede llegar hasta los $50 \mathrm{cmH}_{2} \mathrm{O}$ simulando un suspiro $^{40}$. La ventaja de esta técnica es que el cierre activo de la glotis no es un requisito para su realización. Datos preliminares muestran una mejora en el PFT comparable los dispositivos mecánicos clásicos de asistencia de la tos, con la ventaja que en algunos casos viene incorporado dentro de las opciones del ventilador mecánico ${ }^{40}$.

\section{Conclusión}

Los pacientes con enfermedad neuromuscular tienen un aumento de la morbimortalidad por causa respiratoria ${ }^{15} \mathrm{y}$ una de las causas que contribuye a esta situación es la debilidad de la tos ${ }^{38}$. La eliminación de las secreciones bronquiales depende directamente de la eficacia de la tos ${ }^{3}$. Este reflejo, que además constituye una herramienta terapéutica, se puede evaluar de manera objetiva ${ }^{7,41}$ a través del PFT que depende mayoritariamente del volumen de aire ingresado al sistema respiratorio ${ }^{6}$. Esta evaluación, se puede realizar con dispositivos fáciles de usar y de bajo costo ${ }^{7}$.

A partir del valor obtenido en el PFT y en función de la modalidad ventilatoria, se puede aumentar su eficacia con técnicas de asistencia manual ${ }^{26,30}$ y técnicas de asistencia mecánica ${ }^{16,19}$. El equipo de profesionales encargados del manejo de las secreciones, deberá valorar en cada pacientes y situación, cual es la técnica más adecuada para incrementar la eficacia de la tos.

En la evolución natural de las enfermedades neuromusculares se debe considerar la asistencia de la tos, no sólo con un fin terapéutico, sino que es necesario plantear su utilización con fines preventivos $^{14,15}$, lo que asegurará además de aumentar la sobrevida, mejorar la calidad de vida. Mejorar la efectividad de la tos en pacientes portadores de enfermedades respiratorias con afectación de la musculatura respiratoria es un objetivo primordial que va a impactar en una menor morbimortalidad respiratoria ${ }^{15}$ con la consiguiente menor utilización de recursos sanitarios ${ }^{19}$.

\section{Referencias}

1. Servera E, Sancho J, Zafra M. Tos y enfermedades neuromusculares. Manejo no invasivo de las secreciones respiratorias. Arch Bronconeumol 2003; 39 (9): 418-27.

2. Hess DR. Airway clearance: physiology, pharmacology, techniques, and practice. Respir Care 2007; 1392-6.

3. Chang AB. The physiology of cough. Paediatr Respir Rev 2006; 7 (1): 2-8.

4. Leith DE. The development of cough. Blum BI, Duncan K, editors. Am Rev Respir Dis 1985; 131 (5): S39-S42. 
5. Postiaux G. Fisioterapia respiratoria en el niño. Madrid, España. Editorial McGraw Hill Interamericana; 1999.

6. Trebbia G, Lacombe M, Fermanian C, Falaize L, Lejaille $\mathrm{M}$, Louis A, et al. Cough determinants in patients with neuromuscular disease. Respir Physiol Neurobiol 2005; 146 (2-3): 291-300.

7. Sancho J, Servera E, Díaz J, Marín J. Comparison of peak cough flows measured by pneumotachograph and a portable peak cough flow meter. Am J Phys Med Rehabil 2004; 83 (8): 608-12.

8. Chatwin M, Ross E, Hart N, Nickol AH, Polkey MI, Simonds AK. Cough augmentation with mechanical insufflation/exsufflation in patients with neuromuscular weakness. Eur Respir J 2003; 21 (3): 502-8.

9. Bach JR, Saporito LR. Criteria for extubation and tracheostomy tube removal for patients with ventilatory failure. A different approach to weaning. Chest 1996; 110 (6): 1566-71.

10. Kang SW, Bach JR. Maximum insufflation capacity. Chest 2000; 118 (1): 61-5.

11. Bach JR, Bianchi C, Vidigal-Lopes M, Turi S, Felisari G. Lung inflation by glossopharyngeal breathing and "air stacking" in Duchenne muscular dystrophy. Am J Phys Med Rehabil 2007; 86 (4): 295-300.

12. Bach JR. Mechanical insufflation-exsufflation. Comparison of peak expiratory flows with manually assisted and unassisted coughing techniques. Chest 1993; 104 (5): 1553-62.

13. Anderson J, Hasney K, Beaumont N. Systematic review of techniques to enhance peak cough flow and maintain vital capacity in neuromuscular disease: the case for mechanical insufflation-exsufflation. Phys Ther Rev 2005; 10 (1): 25-33.

14. Bach JR, Ishikawa Y, Kim H. Prevention of pulmonary morbidity for patients with Duchenne muscular dystrophy. Chest 1997; 112 (4): 1024-8.

15. Tzeng AC, Bach JR. Prevention of Pulmonary Morbidity for Patients With Neuromuscular Disease. Chest 2000; 118 (5): 1390-6.

16. Toussaint M, Boitano LJ, Gathot V, Steens M, Soudon P. Limits of effective cough-augmentation techniques in patients with neuromuscular disease. Respir Care. 2009; 54 (3): 359-66.

17. Hull J, Aniapravan R, Chan E, Chatwin M, Forton J, Gallagher J, et al. British Thoracic Society guideline for respiratory management of children with neuromuscular weakness. Thorax 2012; 67 Suppl 1: i1-i40.

18. Estenne M, Heilporn A, Delhez L, Yernault JC, De Troyer A. Chest wall stiffness in patients with chronic respiratory muscle weakness. Am Rev Respir Dis 1983; 128 (6): 1002-7.
19. Chatwin M, Simonds AK. The addition of mechanical insufflation/exsufflation shortens airway-clearance sessions in neuromuscular patients with chest infection. Respir Care 2009; 54 (11): 1473-9.

20. Lyall RA, Donaldson N, Polkey MI, Leigh PN, Moxham J. Respiratory muscle strength and ventilatory failure in amyotrophic lateral sclerosis. Brain 2001; 124 (Pt 10): 2000-13.

21. Kang SW, Kang YS, Sohn HS, Park JH, Moon JH. Respiratory Muscle Strength and Cough Capacity in Patients with Duchenne Muscular Dystrophy. Yonsei Med J 2006; 47 (2): 184-90.

22. Carter GT, Abresch RT, Fowler WM, Johnson ER, Kilmer DD, McDonald CM. Profiles of neuromuscular diseases. Spinal muscular atrophy. Am J Phys Med Rehabil 1995; 74 (5 Suppl): S150-S159.

23. Proctor MR. Spinal cord injury. Anesth Intensive Care 2002; 30 (13): 401-3.

24. Jackson AB, Groomes TE. Incidence of respiratory complications following spinal cord injury. Arch Phys Med Rehabil 1994; 75 (3): 270-5.

25. Schilero GJ, Spungen AM, Bauman WA, Radulovic M, Lesser M. Pulmonary function and spinal cord injury. Respir Physiol Neurobiol 2009; 166 (3): 129-41.

26. Brito MF, Moreira GA, Pradella-Hallinan M, Tufik S. Air stacking and chest compression increase peak cough flow in patients with Duchenne muscular dystrophy. J Bras Pneumol 2009; 35 (10): 973-9.

27. Braun SR, Giovannoni R, O'Connor M. Improving the cough in patients with spinal cord injury. Am J Phys Med 1984; 63 (1): 1-10.

28. Dail CW. "Glossopharyngeal breathing” by paralyzed patients; a preliminary report. Calif Med 1951; 75 (3): 217-8.

29. Nygren-Bonnier M, Wahman K, Lindholm P, Markström A, Westgren N, Klefbeck B. Glossopharyngeal pistoning for lung insufflation in patients with cervical spinal cord injury. Spinal cord 2009; 47 (5): 418-22.

30. Nygren-Bonnier M, Markström A, Lindholm P, Mattsson E, Klefbeck B. Glossopharyngeal pistoning for lung insufflation in children with spinal muscular atrophy type II. Acta Paediatr 2009; 98 (8): 1324-8.

31. Boitano LJ. Equipment options for cough augmentation, ventilation, and noninvasive interfaces in neuromuscular respiratory management. Pediatrics 2009; 123 Suppl 4: S226-30.

32. Torres-Castro R, Vilaro J, Vera-Uribe R, Monge G, Avilés P, Surangy C. Use of air stacking and abdominal compression for cough assistance in people with complete tetraplegia. Spinal Cord (en prensa).

33. Ishikawa Y, Bach JR, Komaroff E, Miura T, Jackson- 
Parekh R. Cough augmentation in Duchenne muscular dystrophy. Am J Phys Med Rehabil 2008; 87 (9): 726-30.

34. Benz E, Villarroel G, Chatwin M, Arellano D, Prado F. Asistencia mecánica de la tos: Dispositivo de tos asistida, in-exsufflator. Neumol Pediatr 2008; 3 (Suppl): 76-82.

35. Barach A, Beck G. Mechanical production of expiratory flow rates surpassing the capacity of human coughing. Am J Med Sci 1953; 226 (3): 241-9.

36. Sivasothy P, Brown L, Smith IE, Shneerson JM. Effects of manually assisted cough and mechanical insufflation on cough flow of normal subjects, patients with chronic obstructive pulmonary disease (COPD), and patients with respiratory muscle weakness. Thorax 2001; 56 (6): 438-44.

37. Gonçalves MR, Honrado T, Winck JC, Paiva JA. Effects of mechanical insufflation-exsufflation in preventing respiratory failure after extubation: a randomized con- trolled trial. Crit Care 2012; 16 (2): R48.

38. Dohna-Schwake C, Ragette R, Teschler H, Voit T, Mellies U. Predictors of severe chest infections in pediatric neuromuscular disorders. Neuromuscul Disord 2006; 16 (5): 325-8.

39. Senent C, Golmard JL, Salachas F, Chiner E, MorelotPanzini C, Meninger V, et al. A comparison of assisted cough techniques in stable patients with severe respiratory insufficiency due to amyotrophic lateral sclerosis. Amyotroph Lateral Scler 2011; 12 (1) 26-32.

40. Geiseler J, Fresenius J, Karg O. Secretion in Patients with Neuromuscular Diseases. Key Major Topics, Technology and Clinical Implications. Humidification in the Intensive Care Unit. $1^{\text {a }}$ ed. Springer; 2012. p. 227-35.

41. Bianchi C, Baiardi P. Cough peak flows: standard values for children and adolescents. Am J Phys Med Rehabil 2008; 87 (6) 461-7. 